\title{
Comparative Investigation of Body Composition in Male Dogs Using CT and Body Fat Analysis Software
}

\author{
Toyokazu KOBAYASHI ${ }^{1)}$, Hiroshi KOIE ${ }^{1) *}$, Akiko KUSUMI ${ }^{1)}$, Masato KITAGAWA ${ }^{2)}$, Kiichi KANAYAMA ${ }^{1)}$ and \\ Kazuya OTSUJI ${ }^{3}$ \\ 1) Laboratory of Veterinary Physiology, College of Bioresource Sciences, Nihon University, 1866 Kameino, Fujisawa, Kanagawa \\ 252-0880, Japan \\ 2)Laboratory of Comprehensive Veterinary Clinical Studies, College of Bioresource Sciences, Nihon University, 1866 Kameino, \\ Fujisawa, Kanagawa 252-0880, Japan \\ 3)Department of Animal Science, Teikyo University of Science, 2-2-1 Senjusakuragi, Adachiku, Tokyo 120-0045, Japan
}

(Received 2 August 2013/Accepted 28 October 2013/Published online in J-STAGE 11 November 2013)

ABSTRACT. In small animal veterinary practices, body condition score (BCS) is generally used to diagnose obesity. However, BCS does not constitute objective data. In this study, we investigated the value of using human body fat analysis software for male dogs. We also compared changes in body fat after neutering. Changes in body fat at the time of neutering (age 1 year) and 1 year later were compared by performing CT scanning and using human body fat analysis software. We found that body fat increased in all the individuals tested. In terms of the site of fat accumulation, subcutaneous fat was more pronounced than visceral fat with a marked increase on the dorsal side of the abdomen rather than the thorax.

KEY WORDS: BCS, body fat analysis software, canine, CT, neutering.

doi: 10.1292/jvms.13-0397; J. Vet. Med. Sci. 76(3): 439-446, 2014

Obesity is defined as the excess accumulation of body fat. It is associated with metabolic problems and directly or indirectly causes a range of endocrine disturbances [25]. In the veterinary field, because obesity is intimately related to the development of type 2 diabetes, hypertension, hyperlipidemia and heart disease, it is regarded as one of the most important issues (risk factors) for health management [8, 23]. Similar to humans, dogs and cats become obese when their calorie intake exceeds their calorie consumption, and obese dogs and cats are reported to have shorter lifespans and a higher incidence of metabolic disorders [25].

In a large clinical study carried out 11 veterinary institutions in the United Kingdom, $21.4 \%$ of dogs were judged to be obese, and $2.9 \%$ were judged to be grossly obese. Therefore, a total of $24 \%$ of 8,268 dogs were found to have exceeded a healthy body weight based on their appearance [6]. In another large-scale study in the United States, data on about 23,000 dogs gathered from 60 animal hospitals showed that $25 \%$ either exceeded a healthy body weight or were judged obese on the basis of their appearance [4]. The U.K. clinical study found that of over 2,000 cats examined by veterinarians, $25 \%$ were either overweight or obese [21]. These study results thus show that around one-quarter of the

*Correspendence to: Koie, H., Laboratory of Veterinary Physiology, College of Bioresource Sciences, Nihon University, 1866 Kameino, Fujisawa, Kanagawa 252-0880, Japan.

e-mail: koie.hiroshi@nihon-u.ac.jp

(C)2014 The Japanese Society of Veterinary Science

This is an open-access article distributed under the terms of the Creative Commons Attribution Non-Commercial No Derivatives (by-ncnd) License $<$ http://creativecommons.org/licenses/by-nc-nd/3.0/ $>$. dogs and cats seen by veterinarians in small-animal practices are either overweight or visibly obese [4, 6, 21].

Neutering via surgical removal of the testes or ovaries in dogs and cats is known empirically to increase the risk of obesity $[7,11,18]$. It has been found that if dogs and cats exceed their ideal body weight by $15 \%$, the harmful physical effects associated with obesity, including heart disease, exacerbation of joint disease and diabetes, become apparent $[4,18]$.

Diagnosis of obesity in dogs is difficult due to the great differences in body type according to breed, age and sex. In clinical practice, body condition score (BCS) and muscle condition score (MCS) are commonly used to determine the degree of obesity. BCS evaluation is a method of assessing body fat that mainly consists of palpation of subcutaneous fat in the thoracic region and evaluation of the degree of obesity on a 5-point or 9-point scale. MCS evaluation is a procedure that comprises visual inspection together with palpation of the temporal bone, scapula, lumbar spine and pelvis [2]. Loss of muscle mass has a negative effect on body strength and immune function and has also been reported to affect mortality in humans $[10,15]$. Determination of MCS in dogs is currently at the development and validation stage. However, determination of the degree of obesity by BCS and MCS methods is affected by the experience and subjectivity of the person making the measurements, and it may not always constitute objective data. BCS is also determined on the basis of palpation and visual inspection, and it is therefore difficult to determine whether visceral fat, which is suggested to be a problem in humans, is present.

In scientific studies, methods used for measuring dog body type have included magnetic resonance imaging (MRI), computed tomography (CT), ultrasound imaging, body wa- 
Table 1. Profiles of the dogs

\begin{tabular}{lccc}
\hline & Breed & Sex & Weight $(\mathrm{kg})$ \\
\hline Case 1 & Airedale terrier & Male & 20.5 \\
Case 2 & Welsh corgi & Male & 10.4 \\
Case 3 & miniature dachshund & Male & 7.3 \\
\hline
\end{tabular}

Table 2. Comparison of amounts of fat and body fat ratios before and after neutering on CT

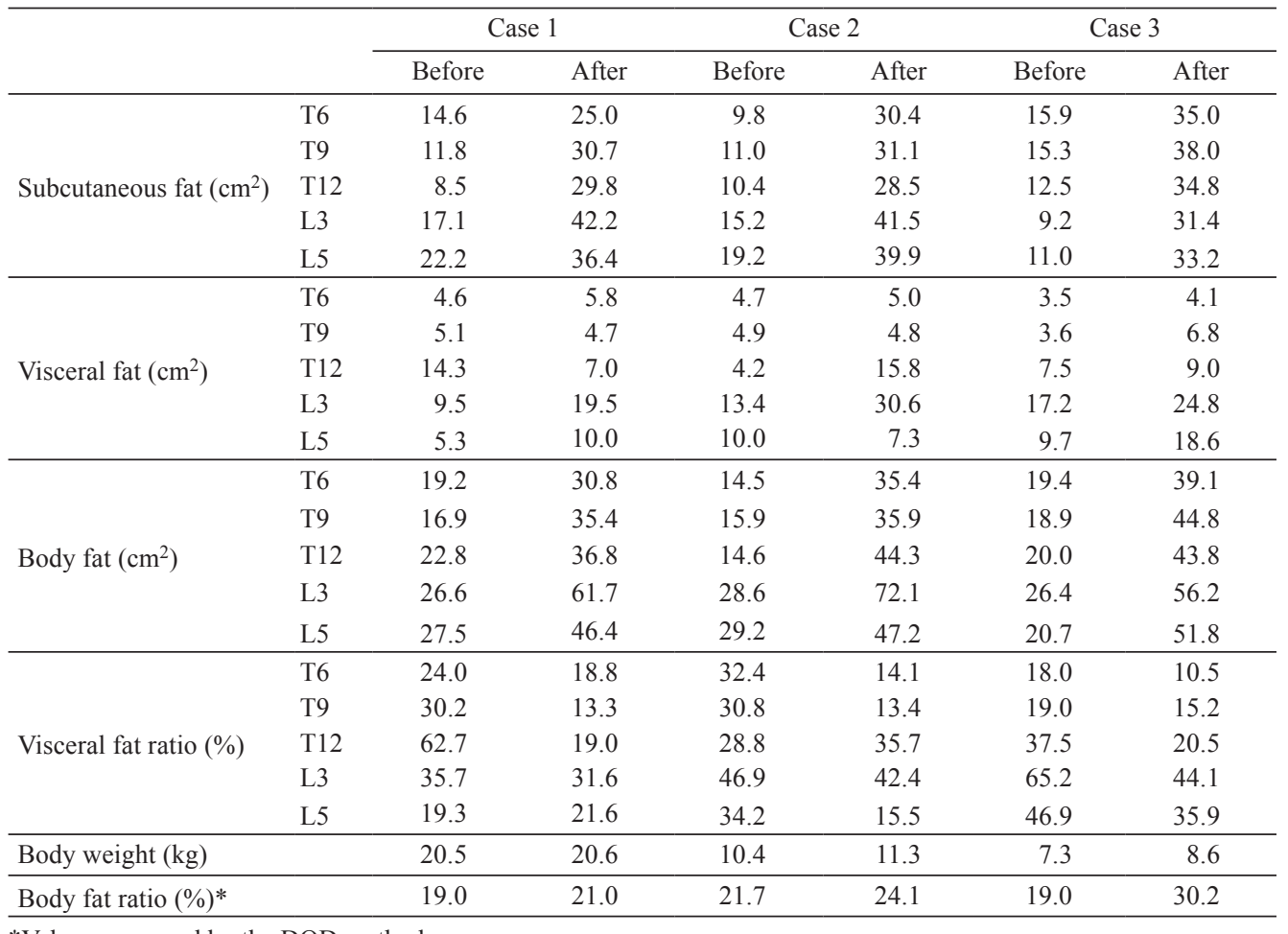

*Values measured by the DOD method.

ter concentration graphs, body water volume measured by isotope dilution, total body potassium, bioelectrical impedance and dual-energy X-ray absorptiometry (DEXA) [14].

In human health care, CT is generally used to measure body fat and body fat ratio, and its value has been established $[13,24]$. In many human hospitals, body fat analyzing software for CT is used during health checkups for metabolic syndrome. In the veterinary clinical field, however, CT is not currently used to measure body fat. No special software for utilizing CT data to measure body fat in dogs has therefore been developed to date. In this study, we investigated the value of using human body fat analysis software for dogs. At the same time, we also focused on the increase in body fat in male dogs after neutering and compared changes in body fat at the time of neutering (age 1 year) and 1 year later by performing CT scanning and using human body fat analysis software.

Animals: The subjects were 3 dogs reared in regular households whose owners requested that they be neutered. Their breeds were miniature dachshund, Welsh corgi and Airedale terrier. All dogs exhibited no abnormal results on regular physical examination, biochemical blood tests and complete blood count testing. All the profiles of the dogs used in this study are shown in Table 1.

All test animals underwent CT scanning and neutering at 1 year of age. They also underwent a further CT scan 1 year after surgery. They were fed 2 types of dog food during the experimental period: Science Diet $(377 \mathrm{kcal} / 100 \mathrm{~g}$; HillsColgate (Japan) Ltd., Tokyo, Japan) or IAMS for adult dogs (363 kcal/100 g; P\&G Japan, Kobe, Japan). The amount of food given was determined by deriving the dogs' resting energy requirement (RER) from their body weight (BW) $\left(\mathrm{RER}=70 \mathrm{BW}^{3 / 4}\right)$ and calculating their energy requirements according to the amount of exercise and living environment (1.6-2.0 RER). The amount of food given to each test animal was fixed during the experimental period.

Anesthetic management: A catheter was placed in the cephalic vein via a $22-24 \mathrm{G}$ cannula in preparation for anesthesia, and a SURFLO plug (Terumo, Tokyo, Japan) was attached. As premedication, after subcutaneous administration of atropine sulfate $(0.025 \mathrm{mg} / \mathrm{kg})$, midazolam hydrochloride $(0.1-0.2 \mathrm{mg} / \mathrm{kg})$ and butorphanol tartrate $(0.1-0.2$ 

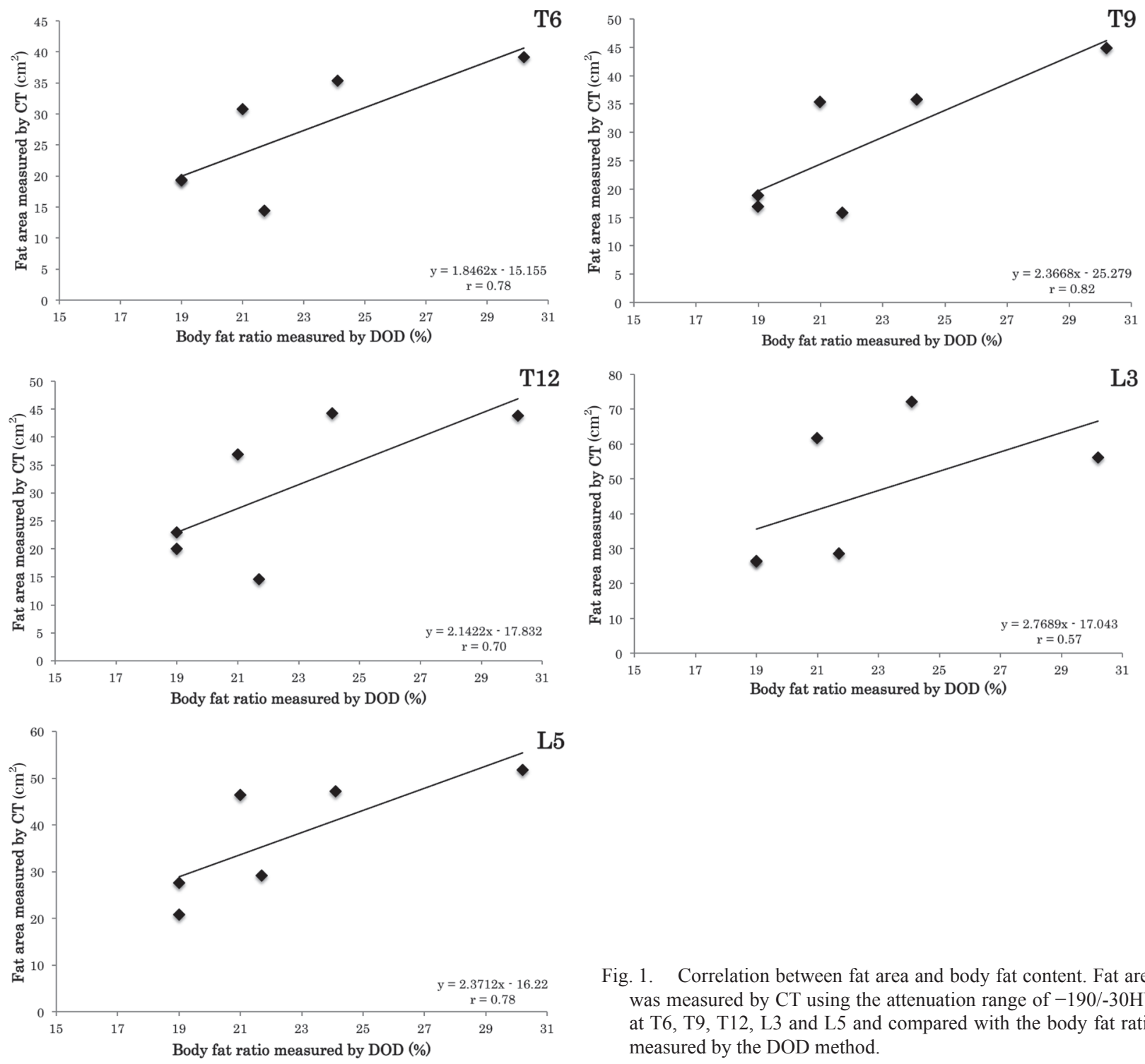

Fig. 1. Correlation between fat area and body fat content. Fat area was measured by CT using the attenuation range of $-190 /-30 \mathrm{HU}$ at T6, T9, T12, L3 and L5 and compared with the body fat ratio measured by the DOD method.

$\mathrm{mg} / \mathrm{kg}$ ) were administered intravenously. After induction of anesthesia with intravenous propofol $(4 \mathrm{mg} / \mathrm{kg})$, dogs were intubated with an endotracheal tube, and anesthesia was maintained by inhalation of isoflurane (1.5\%) and oxygen (2 l/min). Respiratory management during anesthesia was carried out manually, and breath holding by dogs during CT scanning was obtained manually.

The same anesthesia was used during neutering, and respiration was managed by using an automatic artificial ventilator for veterinary use (Compos $\beta$-EV; Metran, Kawaguchi, Japan).

CT imaging conditions: A 16-row multislice CT (Aquilion 16; Toshiba Medical Systems, Otawara, Japan) was used for imaging. Imaging conditions included a tube voltage of 120 $\mathrm{kV}$, tube current of $200 \mathrm{~mA}$ and imaging slice thickness of $1-2 \mathrm{~mm}$. Test animals were held in the prone position during

imaging.

Image processing: Scanned images were reconstructed using the FC10 ORG reconstruction function on the CT scanner, after which they were exported into image processing software (AZE VirtualPlace Plus, AZE, Tokyo, Japan). Image data then underwent final analysis with human body fat analysis software (AZE VirtualPlace Lexus, AZE, Tokyo, Japan), and areas of fat were selected with the CT attenuation value set at $-190 /-30 \mathrm{HU}$ for analysis. We found that, if the analysis settings were set automatically by standard deviation, this software could not be used to obtain appropriate data from dogs. We therefore designated the CT attenuation range indicating areas of fat in humans to select areas of fat. The CT cross-sectional images evaluated in this study were selected from the past studies done [11]. We selected areas from the thoracic (T6, T9 and T12) and lumbar spines (L3 
Before surgery

T6

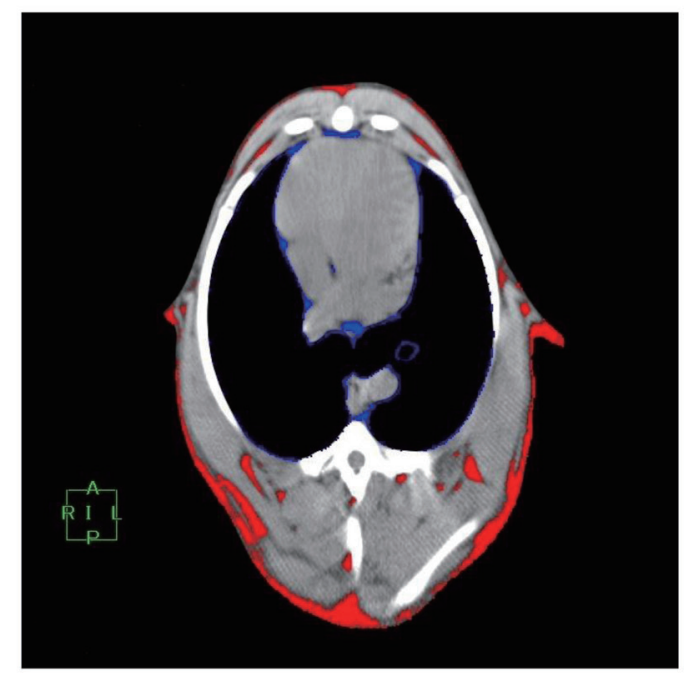

T9

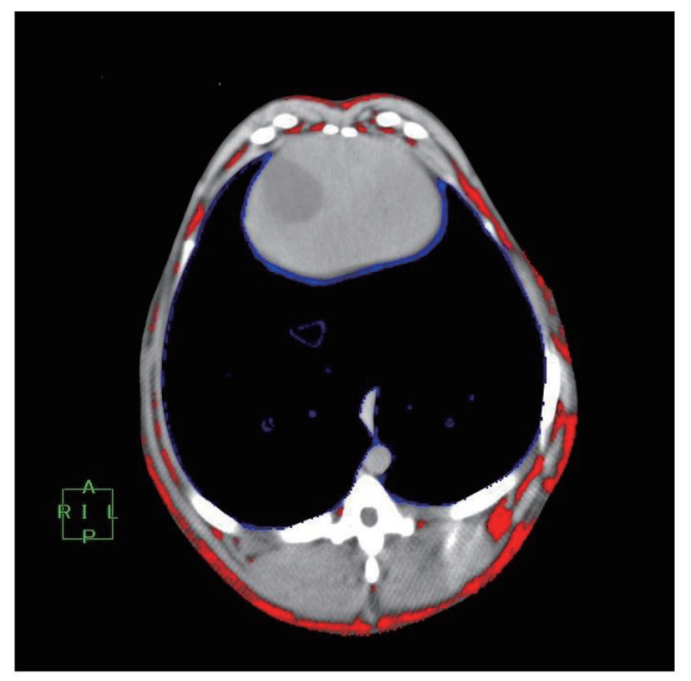

T12

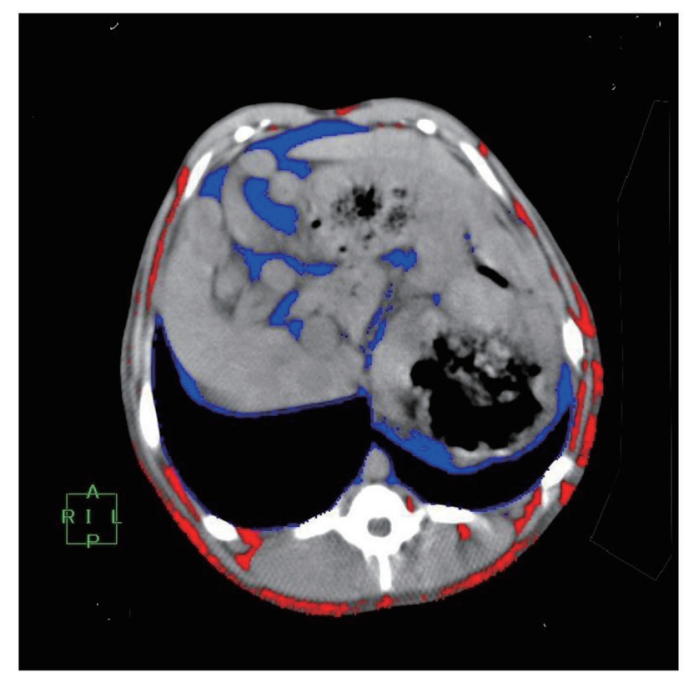

After surgery
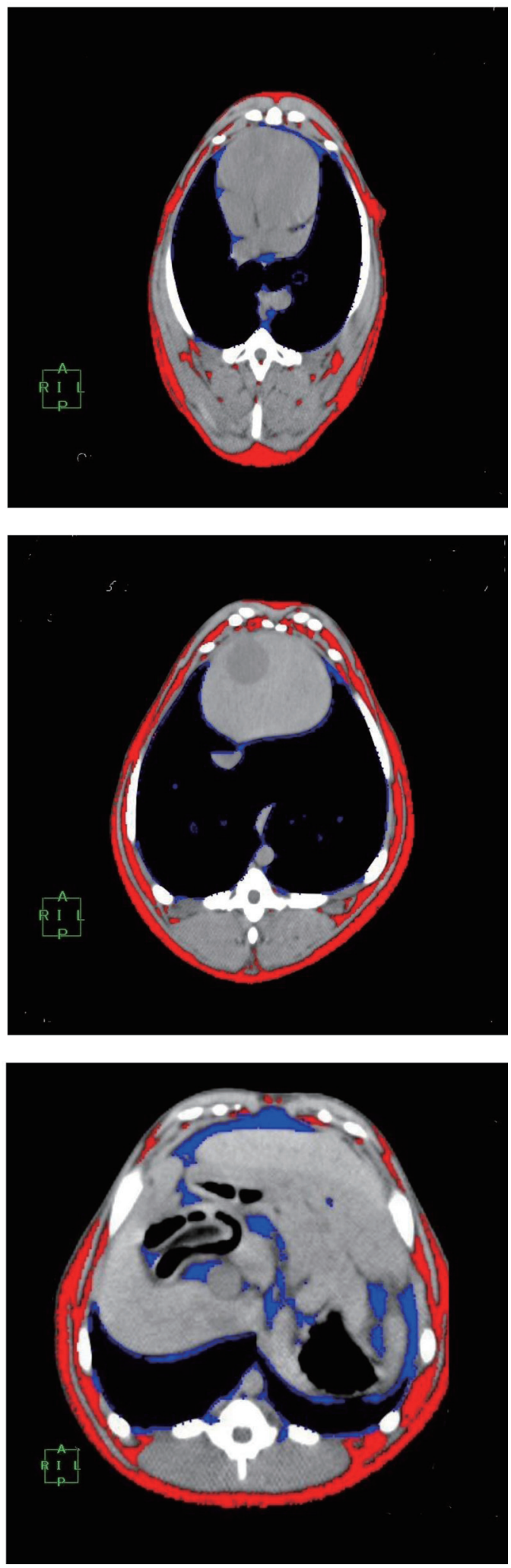

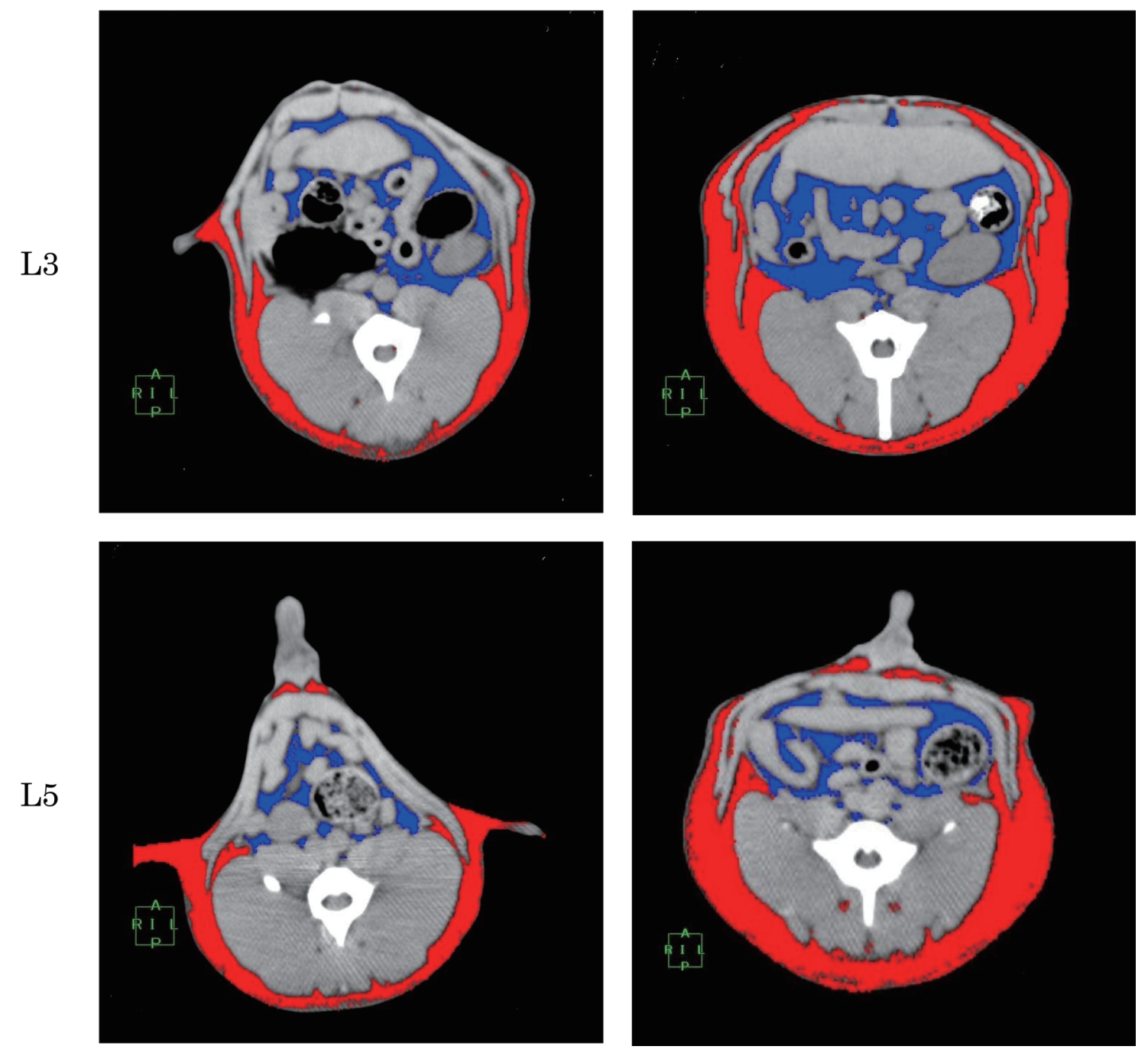

and L5). Changes in amounts of subcutaneous fat and the amount of fat in the abdominal cavity (or thoracic cavity) in each cross section were compared on CT images.

The day before CT scanning was performed, body fat was measured using the deuterium oxide dilution (DOD) method. Deuterium oxide (heavy water, $\mathrm{D}_{2} \mathrm{O}$ ) is a useful label (tracer) because it is evenly distributed throughout body fluids, is not metabolized in vivo and can be measured safely. To measure $\mathrm{D}_{2} \mathrm{O}$, which is always naturally present in blood, blood was sampled from the cephalic vein, and the serum was separated. $\mathrm{D}_{2} \mathrm{O}(0.2 \mathrm{~g} / \mathrm{kg})$ was then administered by subcutaneous injection. Two hr after administration of $\mathrm{D}_{2} \mathrm{O}$, blood was again sampled from the cephalic vein, and serum separation was performed. The concentration of $\mathrm{D}_{2} \mathrm{O}$ was measured using a mass spectrometer (20-22 Isotope
Ratio Mass Spectrometer; Sercon Limited, Cheshire, U.K.). Measurement was done according to Sons' method [17, 22]. Using the measured serum $\mathrm{D}_{2} \mathrm{O}$ concentration and body weight, percentage and amount of body fat were calculated using the equations below.

Amount of body fluids in $\mathrm{kg}=$ body weight $\times 0.2 /$ serum $\mathrm{D}_{2} \mathrm{O}$ density $\% \times 10$

Fat-free mass kg=body fluid / 0.732

Body fat percentage $\%=($ body weight - fat-free mass $) \times$ 100 / body weight

Body fat in $\mathrm{kg}=$ body weight $\times$ body fat percentage

Fig. 2. Comparison between before and after surgery using CT. CT with the attenuation range of $-190 /-30 H U$ was used to measure the fat area at T6, T9, T12, L3 and L5 in Case 1. The red area indicates subcutaneous fat, and the blue area indicates visceral fat. 
Table 3. Comparison of average amounts of fat and body fat ratios before and after neutering on CT

\begin{tabular}{|c|c|c|c|c|}
\hline & & \multicolumn{2}{|c|}{ Average } & \multirow{2}{*}{$t$-test } \\
\hline & & Before & After & \\
\hline \multirow{5}{*}{ Subcutaneous fat $\left(\mathrm{cm}^{2}\right)$} & T6 & 13.4 & 30.1 & $* *$ \\
\hline & T9 & 12.7 & 33.3 & $* *$ \\
\hline & $\mathrm{T} 12$ & 10.5 & 31.0 & $* *$ \\
\hline & L3 & 13.8 & 38.4 & $* *$ \\
\hline & L5 & 17.5 & 36.5 & $* *$ \\
\hline \multirow{5}{*}{ Visceral fat $\left(\mathrm{cm}^{2}\right)$} & T6 & 4.3 & 5.0 & NS \\
\hline & T9 & 4.5 & 5.4 & NS \\
\hline & $\mathrm{T} 12$ & 8.7 & 10.6 & NS \\
\hline & L3 & 13.4 & 25.0 & $* * *$ \\
\hline & L5 & 8.3 & 12.0 & NS \\
\hline \multirow{5}{*}{ Body fat $\left(\mathrm{cm}^{2}\right)$} & T6 & 17.7 & 35.1 & $* *$ \\
\hline & T9 & 17.2 & 38.7 & $* *$ \\
\hline & $\mathrm{T} 12$ & 19.2 & 41.6 & $* *$ \\
\hline & L3 & 27.2 & 63.4 & $* *$ \\
\hline & L5 & 25.8 & 48.5 & $* *$ \\
\hline \multirow{5}{*}{ Visceral fat ratio (\%) } & T6 & 24.3 & 14.2 & $* * *$ \\
\hline & T9 & 26.2 & 14.0 & $* * *$ \\
\hline & $\mathrm{T} 12$ & 45.3 & 25.5 & NS \\
\hline & L3 & 49.3 & 39.4 & NS \\
\hline & L5 & 32.2 & 24.7 & NS \\
\hline Body weight (kg) & & 12.7 & 13.5 & NS \\
\hline Body fat ratio $(\%)^{*}$ & & 19.9 & 25.1 & NS \\
\hline
\end{tabular}

* Values measured by the DOD method. ** $P<0.01$, *** $P<0.05$. ns $P>0.05$.

All dogs examined ( 3 heads) had increased body weights and body fat percentages at 1 year after castration (Table 2). As shown in Table 3, for measurements at cross sections T6, T9, T12, L3 and L5, significant increases in subcutaneous fat accumulation were identified $(P<0.01)$. Also, measurement of the body fat percentage increase using the deuterium oxide dilution method and measurement of the body fat amount using CT were confirmed to have a mutual relationship (Fig. 1).

Visceral fat increased significantly only at the L3 cross section $(P<0.05)$. At the T6 and T9 cross sections, the proportion of fat in the thoracic cavity, as a proportion of the total area of body fat ( $\%$ visceral fat), decreased significantly $(P<0.05)$. At the L3 and L5 cross sections, the proportion of fat in the abdominal cavity, as a proportion of the total area of body fat, decreased overall, but the difference was not significant. These results indicate that the rate of increase in the amount of subcutaneous fat was significantly higher than the rate of increase in the amount of fat in the abdominal and thoracic cavities $(P<0.05)$. The rate of increase in subcutaneous fat at 1 year after neutering was particularly high in the region of the T9, T12 and L3 cross sections at the junction of the thorax and abdomen (Tables 2 and 3). The accumulation of subcutaneous fat was particularly marked on the dorsal side when compared with the ventral side in abdominal cross sections L3 and L5 only (Fig. 2).

In veterinary medicine, the amount of accumulated fat is known to increase after neutering. In livestock animals, such as cows and pigs, for example, castration is known to increase fat accumulation, resulting in softer meat [16]. In our study, we were able to prove that dogs do exhibit increased body fat after castration using $\mathrm{CT}$ and human body fat analysis software. CT image analysis also showed that localization of body fat accumulation has a specific pattern in dogs that differs from that in humans. In humans, fat normally accumulates subcutaneously and in the abdominal cavity due to obesity with accumulation of fat in the abdominal cavity identified as a particular clinical issue [13, 14]. In this study, we found that fat accumulation due to obesity was most marked on the dorsal side of the abdomen in dogs (Fig. 2). Abdominal CT cross-sectional images of all dogs showed that the greatest increase in fat accumulation occurred on the dorsal side of the abdomen when compared with the ventral side.

As shown in Fig. 2, the peritoneal line, which lies on the dorsal side of the abdomen, is not as clearly visualized in dogs as in humans. Human body fat analysis software using CT images generally includes a function that automatically differentiates between fat in the abdominal cavity (visceral fat) and subcutaneous fat. The presence of the peritoneal line on the dorsal side of the abdomen is vital to this process. Due to the fact that the peritoneum on the dorsal side of the abdomen is clearly visualized in humans and the fact that there is a muscle layer between the peritoneum and subcutaneous fat, the body fat analysis software is capable of automatically differentiating between subcutaneous and visceral fat. In dogs, however, the lack of a clear peritoneal line on the dorsal side made it difficult for the software to recognize and 
distinguish between subcutaneous and visceral fat automatically on the dorsal side of the abdomen $[1,5]$. We therefore ended up manually depicting the peritoneal line on the dorsal side of the abdomen. But, as the number of cases we handle increases, we are looking forward to the development of canine software.

In this study, visceral fat, which is regarded as a problem in humans, increased very little when compared with subcutaneous fat in dogs at 1 year after neutering in all the cross sections. The ratio of visceral fat increased in all dogs in only one cross section, L3. When using CT to determine the degree of obesity in humans, judgments are made on the basis of the state of subcutaneous and visceral fat accumulation in abdominal cross sections of the umbilical region [24]. In dogs, the site of the umbilicus varies between different breeds, but generally, it is located around the neighborhood of the L3 cross section. The cross-sectional CT image at L3 was therefore also used in this study to determine the amount of fat in the abdominal cavity.

Other than BCS, no other criteria for determining the degree of obesity in dogs have yet been clearly laid out [2]. As the results of the present study suggested that determination at the L3 cross section may be useful when using CT to determine the degree of obesity, accumulation of visceral fat at this site may provide one index for obesity determination.

We found a high rate of increase in the accumulation of subcutaneous fat in the T9, T12 and L3 cross-sectional fields. In everyday treatment, we primarily use BCS, which depends on visual examination and palpation, to determine the degree of obesity, and the results of the present study showed that palpation of the thorax above the ribs in the T9 and T12 regions may also provide useful information when assessing the BCS. We believe that, in addition to the thoracic region, the state of attachment of subcutaneous fat on the dorsal side of the $\mathrm{L} 3$ region should also be confirmed by palpation, and the BCS should be determined in a comprehensive manner.

According to Ishioka et al. [11], setting dog-specific CT attenuation values rather than using human CT attenuation values enabled them to obtain a correlation between increases in body fat ratio measured by deuterium oxide dilution and CT images. In our study, we used the CT attenuation values generally used to determine the degree of obesity in humans (-190/-30 HU). We were able to obtain full correspondence between the elevations of body fat percentage (Fig. 1). This finding suggests that human body fat analysis software can also be used for dogs.

CT scans are not generally used in veterinary medicine to determine the degree of obesity in dogs. The reasons for this include high imaging costs and the necessity of general anesthesia. In humans, CT, MRI and DEXA are all commonly used to measure the body fat ratio when determining the degree of obesity, and there is a strong correlation between their analytical results $[13,14]$. The use of CT, MRI and DEXA in veterinary medicine has only been reported for cats [3].

When BCS is used to determine the degree of obesity, as is the common practice in veterinary clinical practice, there is no need for special equipment or general anesthesia.
However, it has the disadvantage of being influenced by individual subjectivity and is lacking in objectivity. In our study, all the tested animals showed confirmed increases in visceral fat. However, we were unable to evaluate the change in relation to the change in BCS. In this experiment, we confirmed that the increase in body fat in dogs is most marked on the dorsal side of the abdomen. Normally, palpation of the thoracic rib area is regarded as important for assessing BCS [2]. Our results in this study, however, showed that palpation of the dorsal side of the abdomen should also be emphasized as providing useful information. As the BCS is a method of determining the degree of obesity by palpating the body surface, its results are dependent on the amount of subcutaneous fat. The BCS does not therefore reflect tendencies in visceral fat. In humans, an increase in visceral fat is regarded as a factor that exacerbates lifestyle-related diseases; thus, attention must be paid to the accumulation of visceral fat [23]. The dogs that were the subjects of this study were young (2 years old at the end of the study), and the increase in visceral fat was not as marked as that of subcutaneous fat. However, there was a tendency for visceral fat to increase, particularly in the abdominal region, and it is likely that visceral fat also increases in dogs with advancing age. We believe that future studies of middle-aged and elderly dogs using CT and human body fat analysis software may enable the relationship between visceral fat and lifestyle-related diseases in dogs to be better understood.

In the present study, the body fat ratio was also measured by deuterium oxide dilution. This method showed a gradual increase in body fat ratio in each individual. When the degree of obesity was determined by BCS, the change was not sufficient to produce a change in BCS ranking. CT, however, easily enabled the marked increase in subcutaneous fat on the dorsal side of the abdomen to be determined. The increase in visceral fat in particular was a finding that could not have been identified without the use of CT.

In this study, we neutered dogs at 1 year of age and compared the degree of obesity with that at 1 year later after surgery. According to a report of the Japanese Ministry of Health, Labour and Welfare, human obesity and accumulation of visceral fat are regarded as risk factors for the development of lifestyle-related diseases mainly in middle and old age $[15,19,20]$. Middle age for dogs is generally regarded to start at around age 7 , although this varies with breed. Accumulation of visceral fat may also become more pronounced in dogs in middle and old age, but this has yet to be demonstrated.

We herein proved that CT and human body fat analysis software are also useful in determining the degree of obesity in male dogs.

\section{REFERENCES}

1. Assheuer, J. and Sager, M. 1997. Abdomen. pp. 347-403. In: MRI and CT Atlas of the Dog, Blackwell Science, Berlin.

2. Baldwin, K., Bartges, J., Buffington, T., Freeman, L. M., Grabow, M., Legred, J. and Ostwald, D. Jr. 2010. AAHA Nutritional assessment guidelines for dogs and cats. J. Am. Anim. 
Hosp. Assoc. 46: 285-296. [Medline]

3. Buelund, L. E., Nielsen, D. H., McEvoy, F. J., Svalastoga, E. L. and Bjornvad, C. R. 2011. Measurement of body composition in cats using computed tomography and dual energy X-ray absorptiometry. Vet. Radiol. Ultrasound 52: 179-184. [Medline] [CrossRef]

4. Burkholder, W. J. and Toll, P. W. 2000. Obesity. pp. 401-402. In: Small Animal Clinical Nutrition, 4th ed. (Hand, M. S., Thatcher, C. D., Remillard, R. L. and Roudebush, P. eds.), Mark Morris Institute, Kansas.

5. Done, S. H., Evans, S. A., Goody, P. C., Baines, E. A. and Stickland, N. C. 2009. The abdomen. pp. 324-330. In: Color Atlas of Veterinary Anatomy, vol.3, 2nd ed., Elsevier, Amsterdam.

6. Edney, A. T. and Smith, P. M. 1986. Study of obesity in dogs visiting veterinary practices in the United Kingdom. Vet. Rec. 118: 391-396. [Medline] [CrossRef]

7. Flynn, M. F., Hardie, E. M. and Armstrong, P. J. 1996. Effect of ovariohysterectomy on maintenance energy requirement in cats. J. Am. Vet. Med. Assoc. 209: 1572-1581. [Medline]

8. German, A. J., Ryan, V. H., German, A. C., Wood, I. S. and Trayhurn, P. 2010. Obesity, its associated disorders and the role of inflammatory adipokines in companion animals. Vet. J. 185: 4-9. [Medline] [CrossRef]

9. Ghaem, M. H., Tai, B. C., Wong, T. Y., Tai, E. S., Li, J., Wang, J. J. and Mitchell, P. 2013. Metabolic syndrome and mortality in the elderly: a time-dependent association. Diabetes Res. Clin. Pract. 99: 209-216. [Medline] [CrossRef]

10. Heitmann, B. L. and Frederiksen, P. 2009. Thigh circumference and risk of heart disease and premature death: prospective cohort study. BMJ 339: b3292. [CrossRef]. [Medline]

11. Ishioka, K., Okumura, M., Sagawa, M., Nakadomo, F., Kimura, K. and Saito, M. 2005. Computed tomographic assessment of body fat in beagles. Vet. Radiol. Ultrasound 46: 49-53. [Medline] [CrossRef]

12. Jeusette, I., Detilleux, J., Cuvelier, C., Istasse, L. and Diez, M. 2004. Ad libitum feeding following ovariectomy in female Beagle dogs: effect on maintenance energy requirement and on blood metabolites. J. Anim. Physiol. Anim. Nutr. (Berl) 88: 117-121. [Medline] [CrossRef]

13. Kim, Y. J., Lee, S. H., Kim, T. Y., Park, J. Y., Choi, S. H. and Kim, K. G. 2013. Body fat assessment method using CT images with separation mask algorithm. J. Digit. Imaging. 26: 155-162. [Medline] [CrossRef]

14. Kullberg, J., Brandberg, J., Angelhed, J. E., Frimmel, H., Bergelin, E., Strid, L., Ahlstrom, H., Johansson, L. and Lonn, L. 2009. Whole-body adipose tissue analysis: comparison of MRI, $\mathrm{CT}$ and dual energy X-ray absorptiometery. Br. J. Radiol. 82:
123-130. [Medline] [CrossRef]

15. Landi, F., Cruz-Jentoft, A. J., Liperoti, R., Russo, A., Giovannini, S., Tosato, M., Capoluongo, E., Bernabei, R. and Onder, G. 2013. Sarcopenia and mortality risk in Frail older persons aged 80 years and older: results from ilSIRENTE study. Age Ageing 42: 203-209. [Medline] [CrossRef]

16. Marti, S., Realini, C. E., Bach, A., Pérez-Juan, M. and Devant, M. 2011. Effect of vitamin A restriction on performance and meat quality in finishing Holstein bulls and steers. Meat Sci. 89: 412-418. [Medline] [CrossRef]

17. Mawby, D. I., Bartges, J. W., d'Avignon, A., Laflamme, D. P., Moyers, T. D. and Cottrell, T. 2004. Comparison of various methods for estimating body fat in dogs. J Am Anim Hosp Assoc. Mar-Apr; 40: 109-114.

18. McGreevy, P. D., Thomson, P. C., Pride, C., Fawcett, A., Grassi, T. and Jones, B. 2005. Prevalence of obesity in dogs examined by Australian veterinary practices and the risk factors involved. Vet. Rec. 156: 695-702. [Medline]

19. McNeill, A. M., Katz, R., Girman, C. J., Rosamond, W. D., Wagenknecht, L. E., Barzilay, J. I., Tracy, R. P., Savage, P. J. and Jackson, S. 2006. Metabolic syndrome and cardiovascular disease in older people: The cardiovascular health study A. $J$. Am. Geriatr. Soc. 54: 1317-1324. [Medline] [CrossRef]

20. Ministry of Health Labor and Welfare. National Health and Nutrition Survey 2011 http://www.mhlw.go.jp/bunya/kenkou/ eiyou/dl/h23-houkok u-05.pdf (in Japanese).

21. Scarlett, J. M., Donoghue, S., Saidla, J. and Wills, J. 1994. Overweight cats: prevalence and risk factors. Int. J. Obes. Relat. Metab. Disord. 18 (Suppl. 1): S22-S28. [Medline]

22. Son, H. R., d'Avignon, D. A. and Laflamme, D. P. 1998. Comparison of duel-energy x-ray absorptiometry and measurement of total body water content by deuterium oxide dilution for estimating body composition in dogs. Am. J. Vet. Res. 59: 529-532. [Medline]

23. Tvarijonaviciute, A., Ceron, J. J., Holden, S. L., Cuthbertson, D. J., Biourge, V., Morris, P. J. and German, A. J. 2012. Obesityrelated metabolic dysfunction in dogs: a comparison with human metabolic syndrome. BMC Vet. Res. 8: 147. [Medline] [CrossRef]

24. Yoshizumi, T., Nakamura, T., Yamane, M., Islam, A. H. M. W., Memju, M., Yamasaki, K., Arai, T., Kotani, K., Funahashi, T., Yamashita, S. and Matsuzawa, Y. 1999. Abdominal fat: Standardized technique for measurement at CT. Radiology. 211: 283-286. [Medline] [CrossRef]

25. Zoran, D. L. 2010. Obesity in dogs and cats, a metabolic and endocrine disorder. Vet. Clin. North Am. Small Anim. Pract. 40: 221-239. [Medline] [CrossRef] 